\title{
SATURAÇÃO TEÓRICA EM PESQUISAS QUALITATIVAS: RELATO DE UMA EXPERIÊNCIA DE APLICAÇÃO EM ESTUDO NA ÁREA DE ADMINISTRAÇÃO
}

\section{Theoretical Saturation in Qualitative Research: Report of an Experience of Application under study in the Administration Field}

\author{
Junia Maria Zandonade Falqueto \\ Doutoranda do Programa de Pós- Graduação em Administração. \\ Universidade de Brasília. Brasília, DF. Brasil. \\ e-mail:jufalqueto@gmail.com
}

\section{Valmir Emil Hoffmann}

Doutor em Administração. Professor Titular. Universidade

de Brasília. Brasília, DF. Brasil.e-mail:ehoffmann@unb.br

\author{
Josivania Silva Farias \\ Doutora em Administração. Professor Adjunta. Universidade \\ de Brasília. Brasília, DF. Brasil. e-mail:josivania@unb.br
}

\section{RESUMO}

A transparência e a clareza dos relatórios de pesquisa, destacando a etapa de coleta de dados, são consideradas parâmetros importantes de avaliação do rigor científico em estudos qualitativos. Por essa razão, quanto mais explicitados forem os procedimentos de coleta e análise, mais qualidade poderá ser aferida ao estudo. Um roteiro de saturação teórica empregado na análise dos dados pode colaborar com esse propósito. Este trabalho teve como objetivo relatar uma experiência do uso da técnica de saturação teórica no campo das ciências sociais aplicadas, descrevendo os passos e ressaltando os desafios enfrentados. Foram discutidos e problematizados os seguintes tópicos: categorias de análise, roteiro de pesquisa, critérios para organização da amostra, ponto de saturação, registro e constatação final da saturação teórica. Espera-se, a partir dos resultados alcançados, contribuir para o aprofundamento do conhecimento sobre a técnica, especialmente, entre investigadores qualitativos do campo da Administração. Além disso, o estudo também contribui com a divulgação de um roteiro empírico para sistematização e tratamento de dados em estudos que optarem pela saturação teórica.

Palavras-chave: Pesquisa Qualitativa; Saturação Teórica; Amostragem.

\section{ABSTRACT}

The transparency and clarity of research reports, emphasizing the data collection stage, are considered important parameters for evaluating the scientific rigor in qualitative studies. For this reason, the more explicit the collection and analysis procedures, the more quality can be measured in the study. A theoretical saturation guide used in data analysis can contribute to this purpose. This study aimed to report an experience of using the technique of theoretical saturation in the field of applied social sciences, describing the steps and highlighting the challenges faced. We discussed and problematized the following topics: categories of analysis, research script, criteria for sample organization, saturation point, registration and final verification of the theoretical saturation. We expected, from the results achieved, to contribute to the deepening of the knowledge about the technique, especially among qualitative researchers in the Administration field. In addition, the study also contributes to the dissemination of an empirical guide for the systematization and processing of data in studies that opt for theorical saturation.

Keywords: Theoretical Saturation, Qualitative Research, Sampling. 


\section{INTRODUÇÃO}

Em investigações qualitativas, uma questão frequente é por quanto tempo o pesquisador deve continuar em campo, coletando novos dados (FONTANELLA et al., 2011; SANTOS, 2014; MINAYO, 2017). A regra geral na construção de teorias é coletar informações até que todas as categorias da pesquisa sejam desenvolvidas ou, conforme denominou Strauss e Corbin (2008, p. 205) "estejam saturadas".

A amostragem por saturação é uma ferramenta conceitual que pode ser empregada em investigações qualitativas. É usada para estabelecer o tamanho final de uma amostra, interrompendo a captação de novos dados. Nessa técnica, o número de participantes é operacionalmente definido como a suspensão de inclusão de novos participantes, quando os dados obtidos passam a apresentar, na avaliação do pesquisador, certa redundância ou repetição não sendo considerado produtivo persistir na coleta de dados. Em outras palavras, significa que as informações fornecidas por novos participantes pouco acrescentariam ao material já obtido, não contribuindo de maneira relevante para o aperfeiçoamento da reflexão teórica fundamentada nos dados já coletados (FONTANELLA; TURATO, 2007; FALQUETO, 2012).

Nas palavras dos autores seminais, Glaser e Strauss (2006), por meio dessa técnica constata-se o momento em que se deve interromper a captação de informações pertinentes à discussão de uma determinada categoria de análise no contexto de uma investigação qualitativa. Trata-se, assim, de uma confiança empírica de que a categoria está saturada, levando-se em consideração uma combinação dos seguintes critérios: os limites empíricos dos dados, a integração de tais dados com a teoria (que, por sua vez, tem uma determinada densidade e está diretamente ligada ao referencial teórico) e a sensibilidade teórica de quem analisa os dados. Assim, através da saturação teórica, o pesquisador se torna capaz de designar o momento em que o acréscimo de dados não altera a compreensão do fenômeno. Ou seja, representa um critério que permite estabelecer a validade de um conjunto de observações em pesquisas de caráter qualitativo (GLASER; STRAUSS, 2006).

Dado o interesse crescente sobre o uso da técnica e, até mesmo por este motivo, a técnica necessitar de críticas, aprimoramentos e relatos sobre sua aplicação (O'REILLY; PARKER, 2012), o objetivo deste trabalho é relatar uma experiência de aplicação da saturação teórica, descrevendo os passos e ressaltando os desafios enfrentados em cada etapa. Pretende-se, dessa forma, contribuir para o debate téorico e prático em torno do tema e também divulgar formas de sistematização, de tratamento e análise de evidências empíricas coletadas em pesquisas que utilizem amostras fechadas por saturação, em especial, no campo da Administração.

\section{SATURAÇÃO TEÓRICA}

A forma de constituição de um subconjunto capaz de ser representativo do contexto sob investigação é um importante aspecto de validação em pesquisas científicas, uma vez que os dados a serem analisados emergem fundamentalmente dos participantes que compõem o subconjunto. A formulação de uma amostra transcorre paralelamente a de outros elementos cruciais de validação amostral, tais como: o desenho da pesquisa, o recorte do objeto, a formulação do problema, dos pressupostos ou das hipóteses, a escolha dos instrumentos de coleta de dados e as referências bibliográficas que serão utilizadas na interpretação dos resultados (SANDELOWSKY, 1995; FONTANELLA; RICAS; TURATO, 2008). Em pesquisas quantitativas, o tamanho da amostra pode ser calculado através da estatística. Já nas qualitativas é preciso refletir sobre o que se espera dos participantes do estudo. Para Fontanella, Ricas e Turato (2008), em estudos qualitativos de qualquer natureza, decisões relativas à amostra ocorrerem, em sua maioria, por critérios de seleção que não considerem mensurações das ocorrências estudadas, ao contrário das pesquisas quantitativas que, ao utilizarem amostragem probabilística, não devem prescindir desta caracterização ao calcularem o " $\mathrm{n}$ " adequado aos cálculos estatísticos (SANTOS, 2014; FLICK, 2012; FONTANELLA; RICAS; TURATO, 2008).

Dessa forma, na abordagem qualitativa, a seleção dos participantes não deve seguir critérios quantitativos pelo simples fato de que o objetivo não é quantificar opiniões ou fenomênos, mas explorar uma gama de depoimentos, realizar análise de conteúdo e 
se aprofundar nas relações e nas diferentes representações que um problema pode acarretar (GASKELL, 2000). Conforme afirmam Paiva Junior, Leão e Mello (2011, p. 191), "a objetividade da pesquisa qualitativa é verificada em termos da validade e da confiabilidade de suas observações". Nesse sentido, compreende-se como validade a confiança com que se pode tirar concluções corretas de uma análise e como confiabilidade a consistência com que um procedimento de pesquisa irá analisar um fenomêno da mesma forma em tentativas distintas, se o contexto investigado permanecer constante (PAIVA JUNIOR; LEÃO; MELLO, 2011; BAUER; GAUSER, 2008).

O critério de amostragem por saturação pertence às esferas de validação objetiva e de inferência indutiva e é utilizado para determinar quando o pesquisador deve finalizar o processo de coleta de dados. Tem legitimidade lógica, porém aplica-se somente a casos específicos no âmbito das pesquisas de caráter qualitativo, além de depender da conceitualização precisa das categorias teóricas de análises e das propriedades investigadas (THIRY-CHERQUES, 2009). Ademais, os seus limites não podem, por definição, ser dimensionados a priori. A alternativa de estimação deve ser empírica, fundada na replicação dos experimentos. Assim, o investigador deverá realizar a análise dos dados logo depois de realizada a coleta e só saberá o número de entrevistas (ou observações) que serão necessárias depois de encontrado o ponto em que as categorias saturar ou, em outras palavras, o momento da pesquisa em que não há mais informação relevante para coletar porque os dados estão se repetindo (GLASER; STRAUSS, 2006).

Glaser e Strauss (2006) explicam a saturação como o momento em que o investigador percebe que as lacunas em sua teoria, sobretudo aquelas relacionadas aos conceitos principais, foram em grande parte sanadas, se não completamente. Segundo os autores, o pesquisador deve buscar a saturação de todas as categorias até que fique claro as principais características do fenômeno, caso contrário, pode acabar com um universo vasto de categorias pouco integradas para efeitos de formar um modelo teórico. Nessa visão, o critério de maior relevância para determinar a saturação teórica é formado por uma combinação entre os limites empiricos dos dados, a integração e a densidade da teoria resultante e a sensibilidade teórica do investigador em campo (GLAUSER; STRAUSS, 2006).

Na concepção de Kaufmann (1996), a saturação representa um processo baseado no acúmulo de ideias e conceitos que, à medida que a coleta dos dados avança, tornam-se mais claros e articulados entre si. Nesse processo, as hipóteses de pesquisa se afunilam e formam um núcleo central, ou seja, o modelo teórico atinge maturidade e estabilidade.

Dessa maneira, ao utilizar a amostragem por saturação, é necessário sistematizá-la cuidadosamente. Embora possa parecer um procedimento decorrente de uma constatação facilmente atingível, muitas vezes a averiguação de saturação pode ser realizada de maneira acrítica ou excessivamente subjetiva. $\mathrm{O}$ pesquisador deve estar atento aos critérios utilizados na aplicação da técnica e na constatação da saturação nas categorias (STRATUSS; CORBIN, 2008). Conforme evidenciaram Fontanella, Ricas e Turato (2007), no extremo, o emprego da técnica pode se apoiar apenas no consenso que existe entre pesquisadores qualitativos, sobre a propriedade de utilização deste recurso metodológico, faltando a suficiente discriminação quanto ao seu significado, o que acaba ferindo a transparência da investigação.

Outro ponto é que no emprego da técnica, a coleta e análise de dados são concomitantes, dessa forma, a cada coleta de dados, o pesquisador deve fazer a análise para distinguir quais elementos surgiram e quais foram replicados. É possível encontrar na literatura alguns estudos que ajudam na operacionalização da saturação teórica. Thiry-Cherques (2009) recomenda um mínimo de oito observações, sendo necessárias duas observações depois de encontrado o ponto de saturação para uma confirmação. Esse autor também constatou que, em ciências sociais, o ponto de saturação costuma ocorrer até a $12^{\underline{a}}$ entrevista. Outro estudo publicado sobre o tema é de Guest, Buncem e Johson (2006). Nele, os autores constataram que a saturação ocorre até a $12^{\mathrm{a}}$ entrevista e, além disso, defendem que categorias centrais tendem a aparecer até a $6^{\mathrm{a}}$ entrevista.

Uma variação do uso da técnica foi aplicada por Hoffmann, Belussi, Martínez-Fernández e Reyes Jr.(2016). Naquele trabalho, os autores relataram que havia uma restrição de tempo para a coleta de dados, já que os entrevistados, em sua maioria, estariam 
participando de uma feira comercial, local onde a coleta foi realizada. Assim, decidiram fazer 21 entrevistas sequenciadas, sem a análise concomitante indicada por Glauser e Strauss (2006). De posse das entrevistas, foi feito um sorteio para definir sua ordem de análise. Fazendo assim, a saturação foi alcançada na $11^{\underline{a}}$ entrevista.

\subsection{Apontamentos sobre o uso indiscriminado da Saturação Teórica}

Também são encontrados na literatura estudos que questionam a aplicação indiscrimidada da técnica, sem que haja uma reflexão aprofundada sobre o seu uso e pertinência (ex.: O'REILLY; PARKER, 2012; CAELLI; RAY; MILL, 2003).

No estudo de O'Reilly e Parker (2012) é apresentada uma visão crítica ao argumentarem que é inapropriado impor a saturação a qualquer caso qualitativo. Segundo os autores, cresce o número de estudos em que pesquisadores qualitativos tomam decisões relacionadas à adequação de sua amostra com base apenas na noção de saturação e isso pode acarretar certa confusão na forma como a técnica é compreendida e utilizada no universo científico. Nessa linha, defendem que a saturação teórica é uma técnica mais complexa do que a literatura sugere, que possui uma transparência limitada, uma vez que os dados nunca são verdadeiramente saturados (o pesquisador deve sempre admitir a possibilidade de novas descobertas além da saturação teórica) e corroboram Caelii, Ray e Mill (2003) quando afirmam que a aceitação inquestionável da saturação acaba por perpetuar questões não fundamentadas sobre a adequação da amostragem otimizada e, além disso, comprometem o valor de pesquisas que optam por não utilizar a saturação ou estão em desacordo com seus pressupostos.

De acordo com O'Reilly e Parker (2012), a adequação da amostra não deve ser determinada apenas com base no número de participantes, mas principalmente na adequação dos dados. Os autores citam o exemplo de analistas de discursos que preferem pequenos conjuntos de dados por serem mais apropriados para o seu modo de investigação e citam as obras de Hutchby e Wooffitt (2008) e Have (2007) que permitem fundamentar que há literatura sobre como gerenciar as decisões no processo de pesquisa, usando entrevistas pontuais sem compremeter a qualidade dos resultados.

Nessa linha, o problema da saturação parece surgir quando se admite que a saturação teórica é indicada para qualquer pesquisa qualitativa. A aceitação inquestionável de conceitos como a saturação, para Caelii, Ray e Mill (2003) e O'Reilly e Parker (2012), tornam-se parte de um discurso de qualidade que perpetua mitos sobre a adequação da amostragem e, além disso, minimizam de forma errônea o valor de pesquisas que não estão em acordo com determinados pressupostos relativos à técnica. Conforme afirma O'Reilly e Parker (2012, p. 195): “é possível manter a integridade metodológica dentro de uma tradição particular ao mesmo tempo que se avalia de forma justa outros métodos qualitativos contra suas próprias medidas de qualidade".

Apesar da relevância dos estudos realizados por Caelii, Ray e Mill (2003) e O'Reilly e Parker (2012) e da real necessidade de ampliar o debate sobre o uso da técnica, acredita-se que o fechamento amostral por saturação representa uma alternativa viável para estudos qualitativos nos quais a população não pode ser consultada em sua totalidade, conferindo qualidade empírica e credibilidade às análises dos achados (FONTANELLA; RICAS; TURATO, 2008; STRAUSS; CORBIN, 2008; MINAYO, 2017). Isso é particularmente relevante à pesquisa dado que, em parte considerável dos estudos qualitativos, pesquisadores interrompem a coleta de novos dados devido à limitação de recursos, tais como falta de tempo e de dinheiro, ao invés de se aterem à adequação da amostra e ao rigor ciêntífico (GREEN; THOROGOOD, 2004; O'REILLY; PARKER, 2012). Além disso, há de se considerar que todas as técnicas são limitadas, o que implica em assumir a possibilidade de erros e divergências em relação à saturação teórica.

A partir dessas considerações e com o intuito de promover contribuições práticas e teóricas em relação ao uso da técnica, apresenta-se na próxima seção o relato de uma experiência de aplicação da saturação teórica em um trabalho empírico de natureza qualitativa, realizado na área de Administração. 


\section{RELATO METODOLÓGICO DE UMA EXPERIÊNCIA DE APLICAÇÃO DA SATURAÇÃÓ TEÓRICA}

Inicialmente, cabe frisar o objetivo deste trabalho, tal como apresentado na introdução: relatar uma experiência do uso da técnica de saturação teórica no campo das ciências sociais aplicadas, descrevendo os passos e ressaltando os desafios enfrentados. Para isso, lançou-se mão de um estudo de caso realizado em uma instituição de ensino superior, sendo o objetivo empírico do caso: avaliar a implantação do planejamento em uma organização complexa e de grande porte. Para tanto, a Universidade de Brasilia (UnB), maior universidade pública da região centro-oeste brasileira, foi selecionada como o lócus do estudo.

No caso relatado, a saturação teórica foi empregada para determinar o número de participantes em uma investigação de caráter qualitativo no campo da Administração e o objeto de pesquisa de campo foi o processo de planejamento estratégico da instituição pública de ensino superior brasileira.

As universidades são caracterizadas como organizações complexas, com interesses múltiplos e objetivos difusos (ARAÚJO,1996; MEYER JR., 2008). Isso não ocorre devido somente à sua condição de instituição especializada e acadêmica, mas também pelo fato de executar atividades múltiplas (MEYER JR., 2008), na qual cada tarefa tem uma metodologia de trabalho que lhe é própria. Além disso, a UnB se configura como uma das maiores universidades públicas brasileiras, com mais de 40 mil alunos matriculados e uma comunidade acadêmica que ultrapassa 50 mil pessoas, conforme dados oficiais divulgados no censo da educação superior do País no ano de 2016.

Este trabalho pode ser caracterizado como um estudo de caso exploratório-descritivo, cuja abordagem é qualitativa, situando-se nas soft sciences - por buscar significados, interpretações, em vez dos propósitos exigidos das hard sciences - a mensuração, os testes de hipóteses, as análises causais (DENZIN; LINCOLN, 2006). É possível situar o estudo sob o paradigma interpretativista, em que os significados, o conhecimento ou a compreensão das ações humanas colaboram com a compreensão e, às vezes, até mesmo a construção de teorias (SOARES; ERDMANN, 2013).
Neste caso, o enfoque interpretativista buscou, tão somente, a compreensão e interpretação de significados das falas dos sujeitos, sem, no entanto, aspirar a geração de uma nova teoria.

A população do estudo compreendeu unidades administrativas e acadêmicas da universidade e que estão inseridas no planejamento estratégico da instituição. O número de unidades que participaram das entrevistas foi definido por meio da saturação teórica, uma vez que seria difícil entrevistar a todos devido ao número de setores, que ultrapassam 70 unidades. Os gestores entrevistados foram escolhidos a partir do cargo que exerciam à epoca da coleta de dados, do conhecimento prático que possuem e da competência para atuar no planejamento da unidade administrativa ou acadêmica à qual estava vinculado, conforme registro da organização. Para determinar a ordem das unidades participantes do estudo, realizou-se um sorteio, considerando as características de uma amostra probabilística estratificada, ou seja, na qual a população é composta por estratos bem definido e cuja possibilidade de participação é a mesma para cada unidade (RICHARDSON, 1999). Assim, separaram-se as unidades administrativas e acadêmicas, obtendo-se duas listas: uma para as unidades administrativas e outra para as acadêmicas, conforme detalhado no passo 3 do roteiro apresentado neste trabalho. As entrevistas ocorreram de forma intercalada entre as unidades acadêmicas e administrativas, respeitando-se a ordem do sorteio. Esse critério foi aplicado para evitar que um dos estratos pudesse ter peso maior nos resultados.

Quanto ao cargo e à função dos respondentes, tomou-se o devido cuidado para se entrevistar apenas gestores que participassem ativamente das etapas de planejamento, de forma que a amostra fosse a mais qualificada possível, pois, conforme afirma Neves (1996), em estudos qualitativos, os resultados encontrados dependerão em alto grau da perspectiva dos participantes da situação estudada. Dos dezesseis que foram entrevistados, nove são professores que exerciam cargos administrativos e sete são funcionáriosadministrativos da organização. Participaram da pesquisa gestores de nove unidades administrativas e de sete unidades acadêmicas.

Por fim, admitindo que só haja saturação teórica quando o pesquisador chega à conclusão de 
que a interação entre as atividades de pesquisa de campo (por meio da coleta de novos elementos) e a percepção do investigador (por meio da codificação dos dados coletados) não mais fornecem elementos para balizar ou aprofundar a teorização (GLAUSER; STRAUSS, 2006), foi realizada a sequência de seis passos para a constatação da saturação teórica, conforme detalhado a seguir.

\section{PASSO 1: DEFINIÇÃO DAS CATEGORIAS DE ANÁLISE}

O primeiro passo diz respeito à determinação das categorias de análise, quando se devem determinar quais os conceitos que melhor representam o fenômeno e que serão investigados a partir do processo de amostragem por saturação. Categorias são conceitos derivados dos dados ou da literatura, que representam o fenômeno, o objeto que está sob investigação. Definir uma categoria significa classificar um conceito e essa classificação implica, explicita ou implicitamente, em uma ação a ser tomada em relação ao objeto investigado (STRAUSS; CORBIN, 2008).

Sobre a escolha das categorias, o nome deve ser gráfico o suficiente para lembrar rapidamente o pesquisador do seu referente. Como categorias representam fenômenos, elas devem ser diferenciadas conforme a perspectiva de análise, o foco e o contexto da pesquisa. Os conceitos, ao serem categorizados, tornam-se propriedades ou descritores do objeto (STRAUSS; CORBIN, 2008). Para exemplificar, Strauss e Corbin (2008, p. 115) escrevem: "um analista poderia rotular pássaros, planos e pipas como 'voô', enquanto outro poderia rotulá-los como 'instrumentos de guerra' porque o contexto é diferente".

É possível utilizar a saturação tanto com categorias definidas a priori como a posteriori, o que se deve ter em mente é que, o ponto exato de saturação será sempre a posteriori, por definição. No caso discutido, foram investigadas duas categorias pela técnica de saturação teórica, ambas definidas a partir da teoria: barreiras à implantação do planejamento estratégico; e eficácia da implantação do planejamento estratégico.

Nessa etapa, o principal enfrentamento é o de selecionar, com base nas informações encontradas na teoria ou em campo, elementos que melhor representem o objeto da pesquisa. Ao categorizar, reduzem-se grandes quantitades de dados a bloco de dados que são mais simples de administrar e relacionar (POZZEBON; FREITAS; PETRINI, 1997; STRAUSS; CORBIN, 2008).

O processo de amostragem teórica pode propiciar ao pesquisador a emersão de categorias ainda não moldadas pela literatura. Isso é bastante útil, por exemplo, em estudos cuja abordagem é a Grounded Theory, em que o objetivo da categorização (a posteriori) é gerar teoria e conhecimento sobre um fenômeno anteriormente desconhecido, isto é, a "descoberta de teorias a partir de dados coletados sistematicamente" (PETRINI; POZZEBON, 2009, p.2). Por outro lado, como é o caso deste estudo, a amostragem teórica também pode ser usada na realização de comparações entre os achados, fatos, percepções, locais, pessoas etc. e os conceitos pré-existentes, visando encontrar variações entre conceitos e fenômeno, bem como buscando tornar densas as categorias definidas a priori (STRAUSS; CORBIN, 2008). Portanto, o propósito deste estudo é mais modesto quando comparado àquele previsto em esforços voltados ao desenvolvimento de teorias, como bem representado na Grounded Theory.

Essa postura encontra respaldo em Minayo (2017) quando justifica sua escolha de não optar apenas por um ou outro procedimento (categorizaçaõ $a$ priori ou somente a posteriori). Os dados, portanto, não só ajudam a construir teorias, como também a compreender ou adensar aquelas já existentes.

Todos os achados empíricos iam sendo confrontados com uma vasta literatura que, a partir de uma revisão inicial, fui descobrindo e dela me apossando durante e depois do estudo empírico. As questões surgidas no campo me ajudaram a entender as referências teóricas e vice-versa. Caso me perguntem se esgotei todas as possibilidades e se houve uma saturação na compreensão do objeto, direi que não. Mas, tenho certeza de que me aproximei bastante do objeto e consegui mostrar como o tema é complexo (p.7). 


\section{PASSO 2: DEFINIÇÃO DO ROTEIRO DE PESQUISA}

Com as categorias definidas, o próximo passo requereu a construção de um roteiro de perguntas. A saturação também pode ser utilizada em outras formas de se coletar dados, como observação participante, grupo focal, diários de pesquisa e análise de dados de ocorrência natural, porém é mais comu- mente empregada com o uso de entrevistas e roteiros semiestruturados (THIRY-CHERQUES, 2009; O 'REILLY; PARKER, 2012). Esse esquema facilita o processo para encontrar o ponto de saturação, quando as categorias saturam e novas informações não são mais encontradas, uma vez que cabe ao pesquisador identificar os elementos em cada resposta e verificar repetições (Ver Quadro 1).

Quadro 1 Roteiro das entrevistas conforme categoria investigada

\begin{tabular}{l|l}
\hline \multicolumn{1}{c|}{ Categoria } & \multicolumn{1}{c}{ Perguntas constantes do Roteiro de Entrevistas } \\
\hline $\begin{array}{l}\text { Barreiras à implantação do } \\
\text { planejamento estratégico }\end{array}$ & $\begin{array}{l}\text { 1. Como o planejamento estratégico (PE) é utilizado nas decisões da sua unidade? } \\
\text { 2. Quais são as barreiras à implantação do PE? Dê exemplos. } \\
\text { 3. Quais são os facilitadores à implantação do PE ? Dê exemplos. } \\
\text { 4. Que sugestões teria para o aperfeiçoamento da implantação do PE na Universidade? }\end{array}$ \\
\hline $\begin{array}{l}\text { Eficácia da implantação do } \\
\text { planejamento estratégico } \\
\text { na instituição }\end{array}$ & $\begin{array}{l}\text { 5. Como o PE é utilizado nas decisões da sua unidade? } \\
\text { 6. Como percebe a relação entre o objetivo previsto e o resultado obtido na sua Unidade? } \\
\text { 7. Na sua percepção, todos os objetivos planejados são implantados? O que o leva a pensar assim? } \\
\text { 8. Há objetivos que são implantados que não foram planejados? O que o leva a pensar assim? } \\
\text { 9. Como avalia a implantação do PE na sua unidade? O que o leva a pensar assim? }\end{array}$ \\
\hline
\end{tabular}

Fonte: elaborado pelos autores.

A maior parte das dificuldades ao se utilizar a técnica de saturação teórica resulta de erros na formulação dos quesitos e da má construção do protocolo da pesquisa. Sobre esse aspecto, Thiry-Cherques (2009) recomenda que as questões do roteiro sejam construídas de forma a se evitar três erros: a formulação dúbia dos quesitos; a grande amplitude de respostas; e o alto grau de variabilidade de diferenciação nas respostas. Essas providências ajudam a reduzir o número de observações necessárias ao alcance da saturação, uma vez que facilitam o próximo passo - a codificação das categorias.

No curso da investigação a que este trabalho faz referência, ocorreram dificuldades de se obterem respostas condizentes com os objetivos traçados para determinada pergunta. Formuladas de maneiras diretas 'quais as barreiras à implantação do planejamento estratégico?' e ' quais os facilitadores à implantação do planejamento estratégico?' constatou-se que, para a maioria dos entrevistados, as barreiras confundem-se com os facilitadores de tal forma que um facilitador se traduz em medidas de combate às barreiras identificadas, tornando a pergunta desnecessária.
Para evitar problemas dessa natureza, parece pertinente elaborar roteiros com perguntas objetivas e focadas, de forma a evitar grandes amplitudes de respostas, e que estejam previstos testes que possibilitem repensar as questões. Conforme Duarte (2002), muitos problemas podem ser identificados no roteiro das entrevistas quando elas saem do papel e ganham significado na interação entrevistador/entrevistado. Por essa razão, o roteiro deve ser um instrumento flexível para orientar a condução da entrevista, e precisa ser revisto para que se possa avaliar se atende os objetivos definidos para a investigação.

\section{PASSO 3: DEFINIÇÃO DE CRITÉRIOS PARA A ORGANIZAÇÃO DA AMOSTRA}

Essa é a etapa na qual são definidos critérios de ordem para os participantes do estudo. Ao optar pelo uso da saturação teórica, sugere-se que os possíveis participantes da pesquisa sejam organizados conforme critérios claros, que garantam a mesma possibilidade de participação para qualquer indíviduo que 
cumpra os requisitos do perfil estabelecido, evitando qualquer viés na determinação dos participantes e na ordem das entrevistas.

Conforme explicita Minayo (2017), informações prestadas por indivíduos implicados em determinado objeto de pesquisa representam um conjunto maior, quando observadas algumas precondições. De acordo com a autora, uma entrevista com alguém de um grupo pode ser compreendida, ao mesmo tempo, como um depoimento pessoal e coletivo. Esse argumento é particularmente importante quando se trata da saturação teórica. Primeiro, porque ressalta a importâcia da definição clara dos indivíduos que poderão participar do estudo. É relevante que todos estejam vinculados à dimensão do fenômeno investigado da forma mais profunda possível e, por isso, precisam ser selecionados com atenção. Assim, o pesquisador deve privilegiar aqueles que detêm características predeterminadas e de interesse da investigação, e jamais permitir que a amostra seja formada por elementos "soltos no conjunto da proposta qualitativa" (MINAYO, 2017, p. 4). Segundo, porque corrobora a premissa fundamental da saturação teórica na qual se admite que haja um número suficiente de interlecutores capaz de fornecer os dados necessários para a comprensão de um fenômeno (GLASER; STRAUSS, 2008; MINAYO, 2017).

O pesquisador deve ter em mente que o tamanho amostral na saturação considera o número de sujeitos suficientes conforme ocorram reincidências nas informações coletadas - sem que sejam desprezadas informações relevantes para a compreensão do fenômeno. Assim, a amostra ideal é aquela que possibilita refletir a totalidade da população em suas múltiplas dimensões. O critério da amostragem por saturação não é numérico, tampouco definido por conveniência. Na saturação teórica, busca-se organizar a amostra e, então, realizar a coleta de dados com o número de sujeitos capaz de refletir a realidade do objeto investigado, em todas as suas dimensões (MINAYO, 1999). Dessa forma, torna-se também relevante, além de determinar o perfil da amostra com clareza, estabelecer uma ordem para a coleta de informações, dado que nem todos os indivíduos aptos para participar do estudo serão consultados.

No estudo em pauta, após a definição das características necessárias para participação no estudo, optou-se pela realização de um sorteito para determinar a ordem em que as entrevistas seriam realizadas. A estrutura da organização conta com duas unidades distintas entre si. A primeira delas, diretamente relacionada com a área fim da instituição de ensino, são as unidades acadêmicas. A segunda, responsáveis pela manutenção e funcionamento administrativo da universidade, são as unidades administrativas. Separaram-se as unidades administrativas e acadêmicas e realizou-se um sorteio para cada segmento, obtendo-se assim duas listas: uma para as unidades administrativas e outra para as unidades acadêmicas. As entrevistas ocorreram de forma intercalada entre as listas, respeitando a ordem do sorteio. Com esse critério, evitou-se que um dos estratos pudesse ter um peso maior nos resultados do estudo. Indivíduos que não manifestaram interesse em participar da pesquisa, ou deixaram de participar por algum empecilho, foram substituídos pela unidade sequencial, sem prejuízo à ordem determinada pelo sorteio. A seguir, apresenta-se, a título de exemplificação, uma das listas ordenada, no caso, a lista das unidades acadêmicas. As entrevistas foram identificadas conforme a ordem de realização, considerando que houve revesamento entre unidades acadêmicas e administrativas. Nesse caso, o primeiro entrevistado referente às unidades acadêmicas foi um representante da unidade Faculdade de Educação, e correspondeu à segunda entrevista realizada no estudo (E2), sendo que a primeira (E1) foi realizada entre as unidades administrativas e consta de lista própria das unidades administrativas, semelhante à apresentada no Quadro 2.

Quadro2 Lista de unidades acadêmicas ordenadas conforme sorteio realizado para definição da sequência das entrevistas

\begin{tabular}{l|c}
\multicolumn{1}{c|}{$\begin{array}{c}\text { Unidades Acadêmicas } \\
\text { Ordem sorteada para entrevistas }\end{array}$} & $\begin{array}{c}\text { Identifi- } \\
\text { cação da } \\
\text { entrevista }\end{array}$ \\
\hline Faculdade de Educação (FE) & E2 \\
\hline Instituto de História (IH) & E4 \\
\hline Faculdade de Comunicação (FAC) & E6 \\
\hline Faculdade de Educação Física (FEF) & cancelada \\
\hline Instituto de Ciências Biológicas (IB) & E8 \\
\hline Faculdade de Economia, Administração e & E10 \\
Contabilidade (Face) & \\
\hline
\end{tabular}




\begin{tabular}{|c|c|}
\hline $\begin{array}{l}\text { Unidades Acadêmicas } \\
\text { Ordem sorteada para entrevistas }\end{array}$ & $\begin{array}{l}\text { Identifi- } \\
\text { cação da } \\
\text { entrevista }\end{array}$ \\
\hline Instituto de Química (IQ) & E13 \\
\hline Instituto de Ciências Exatas (IE) & cancelada \\
\hline Instituto de Psicologia (IP) & E15 \\
\hline $\begin{array}{l}\text { Faculdade de Agronomia e } \\
\text { Medicina Veterinária (FAV) }\end{array}$ & $\begin{array}{c}\text { Ponto de } \\
\text { saturação } \\
\text { encontrado }\end{array}$ \\
\hline \multicolumn{2}{|l|}{ Faculdade de Arquitetura e Urbanismo (FAU) } \\
\hline \multicolumn{2}{|l|}{ Fazenda Água Limpa (FAL) } \\
\hline \multicolumn{2}{|l|}{ Instituto de Ciências Políticas (IPOL) } \\
\hline \multicolumn{2}{|l|}{ Faculdade UnB Planaltina (FUP) } \\
\hline \multicolumn{2}{|l|}{ Instituto de Relações Internacionais (IREL) } \\
\hline \multicolumn{2}{|l|}{ Faculdade de Direito (FD) } \\
\hline \multicolumn{2}{|l|}{ Faculdade de Medicina (FM) } \\
\hline \multicolumn{2}{|l|}{ Faculdade de Tecnologia (FT) } \\
\hline \multicolumn{2}{|l|}{ Faculdade de Ciências da Informação (FCI) } \\
\hline \multicolumn{2}{|l|}{ Instituto de Letras (IL) } \\
\hline \multicolumn{2}{|l|}{ Faculdade UnB Gama (FGA) } \\
\hline \multicolumn{2}{|l|}{ Instituto de Geociências (IG) } \\
\hline \multicolumn{2}{|l|}{ Instituto de Artes (IDA) } \\
\hline \multicolumn{2}{|l|}{ Faculdade de Saúde (FS) } \\
\hline \multicolumn{2}{|l|}{ Instituto de Ciências Sociais (ICS) } \\
\hline \multicolumn{2}{|l|}{ Instituto de Ciência Política (IPOL) } \\
\hline Faculdade de Medicina (FM) & \\
\hline
\end{tabular}

Fonte: elaborado pelos autores.

\section{PASSO 4: LEVANTAMEN- TO DE ELEMENTOS NOVOS VERSUS ELEMENTOS CONFIR- MADOS EM CADA COLETA}

Essa etapa envolve a codificação dos dados coletados e ocorre após a realização de cada entrevista. Ou seja, envolve um processo analítico, por meio do qual os conceitos são identificados e suas propriedades e dimensões são descobertas nos dados coletados
(STRAUSS; CORBIN, 2008). Trata-se de levantar os elementos (subcategorias) que são relevantes para o objeto de estudo e classificá-los conforme suas características. O objetivo, segundo Corbin (2003), é levar os dados brutos a níveis mais amplos, de modo que permitam posteriormente a discussão das características relevantes do conteúdo.

Nessa etapa, o pesquisador deve explorar individualmente cada entrevista antes de partir para a próxima. Trata-se de uma etapa individual, na qual se abre um leque de informações. O maior desafio dessa fase é o fato de que, por se tratar de uma etapa exaustiva, na saturação teórica, precisa ser feita logo após a coleta dos dados. Ou seja, após cada entrevista, o pesquisador precisa tratar os dados para reconhecer elementos novos e elementos que já foram citados anteriormente por outros entrevistados.

Recomenda-se que sejam apurados com muita atenção os elementos (subcategorias relacionadas com as categorias definidas a priori para análise) que forem repetidos, ou confirmados, e os novos elementos. Isso é relevante, pois tal distinção propiciará o encontro do ponto de saturação das categorias investigadas. Essa etapa deve ser detalhada com clareza pelo pesquisador, uma vez que é fundamental para que o leitor constate como foi encontrado o ponto de saturação. Na investigação aqui relatada, a organização foi feita por quadros, que diferenciam elementos confirmados e novos, conforme representado nos Quadros 3, 4 e 5.

Quadro 3 Análise da entrevista 1 com identificação dos primeiros elementos

\begin{tabular}{l} 
Entrevista 1 \\
$\begin{array}{c}\text { Identificação dos primeiros elementos } \\
\text { Categorias Barreiras e Facilitadores à implantação do } \\
\text { Planejamento Estratégico na Instituição }\end{array}$ \\
\hline 1. Problemas com a comunicação interna \\
\hline 2. Falta de participação na elaboração do Planejamento Estratégico \\
\hline 3. Falta de recursos tecnológicos \\
\hline 4. Falta de cultura de planejamento \\
\hline 5. Docentes não preparados para atuar em cargos administrativos \\
\hline 6. Falta de apoio da Alta Gestão
\end{tabular}

Fonte: elaborado pelos autores. 
Quadro 4 Análise da entrevista 2 com inclusão de novos elementos e confirmação daqueles já levantados

\section{Entrevista 2}

Confirmou 4 elementos e incluiu 3

Categorias Barreiras e Facilitadores

CONFIRMOU

1. Problemas com a comunicação interna

2. Falta de participação na elaboração do Planejamento Estratégico

3. Falta de recursos tecnológicos

5. Docentes não preparados para atuar em cargos administrativos

INCLUIU

7. Alta rotatividade de pessoal administrativo

8. Baixo nível de qualificação dos corpo técnico-administrativo

9. Falta de articulação entre o planejamento e o orçamento

Fonte: Elaborado a partir de FALQUETO (2012).

Quadro 5 Análise da entrevista 3 com inclusão de novos elementos e confirmação daqueles já levantados

\section{Entrevista 3}

Confirmou 4 elementos e incluiu 1 Categorias Barreiras e Facilitadores

CONFIRMOU

2. Falta de participação na elaboração do Planejamento Estratégico

3. Falta de recursos tecnológicos

4. Falta de cultura de planejamento

9. Falta de articulação entre o planejamento e o orçamento

INCLUIU

10. Alta interdependência entre as unidades

Fonte: Elaborado pelos autores.

Tabela 1 Saturação teórica das respostas coletadas

\begin{tabular}{|c|c|c|c|c|c|c|c|c|c|c|c|c|c|c|c|c|}
\hline & \multicolumn{16}{|c|}{ Entrevistas } \\
\hline Categorias & $\mathrm{E} 1$ & E2 & E3 & E4 & E5 & E6 & E7 & E8 & E9 & E10 & E11 & E12 & E13 & E14 & E15 & E16 \\
\hline Barreiras & 1 & 1 & 1 & 1 & 0 & 1 & 0 & 1 & 0 & 1 & 1 & 0 & 0 & 0 & 0 & 0 \\
\hline Eficácia & 1 & 1 & 1 & 1 & 1 & 0 & 0 & 1 & 0 & 0 & 0 & 0 & 0 & 0 & 0 & 0 \\
\hline
\end{tabular}

Fonte: elaborado pelos autores.

\section{PASSO 5: REGISTRO EM UMA TABELA DO QUE FOI ENCON- TRADO EM CADA COLETA}

Trata-se da construção de uma representação gráfica que permita a visualização dos elementos analíticos levantados nas entrevistas. A Tabela 1 é a exemplificação de como essa representação foi feita na pesquisa original. Da primeira linha da tabela, constam todas as entrevistas realizadas numeradas conforme a ordem cronológica. E nas colunas constam as categorias que foram investigadas. Nas linhas 2 e 3 foram atribuídos o valor de 1 (um) para informar que há, pelo menos, uma nova informação e 0 (zero) para indicar que não foi encontrada nenhuma nova informação na respectiva entrevista.

Essa etapa facilita a compreensão de como o ponto de saturação é encontrado. Ao visualizar graficamente a constatação de novas informações ou repetições de informações já coletadas, o pesquisador passa a ter maior clareza visual (FONTANELLA et al., 2011). Dessa forma, representar graficamente o ponto de alcance da saturação teórica é exercício simples, sem grandes enfrentamentos, que facilita a compreensão e o controle no uso da técnica. 


\section{PASSO 6: CONFIRMAÇÃO DA SATURAÇÃO EM CADA CA- TEGORIA E PRINCIPAIS DIFI- CULDADES ENFRENTADAS}

Esta é a última etapa para constatação da saturação teórica. Ao observar a Tabela 1, verifica-se que, para a categoria Eficácia, o ponto de saturação ocorreu na entrevista 9 (E9). Entretanto, as entrevistas continuaram porque a categoria Barreiras ainda não havia atingido a saturação. Nesse exemplo, a saturação teórica de ambas as categorias foi considerada como tendo ocorrido na entrevista de número 12 (E12), ou seja, a partir desse ponto nenhuma nova informação foi identificada e considerada relevante para a teorização. Após essa constatação, novas entrevistas foram realizadas para a necessária confirmação. Thiry-Cherques (2009) recomenda que sejam feitas duas entrevistas adicionais depois de encontrado o ponto de saturação. No estudo relatado, optou-se por realizar mais quatro entrevistas, a fim de se ter uma margem maior de segurança em relação à saturação.
Uma sugestão, com base nesta experiência vivenciada, é que investigadores qualitativos, ao optarem pela saturação teórica, apoiem-se em experiências anteriores. O fato de ir a campo sem ter ideia do número de coletas que serão necessárias até a constatação do ponto de saturação gera, muitas vezes, insegurança e pode representar um empecilho ao uso da técnica. Por outro lado, é possível encontrar na literatura alguns trabalhos que ajudam a minimizar esse sentimento. Guess, Bunce e Johnson (2006) e Thiry-Cherques (2009) realizaram estudos e comprovaram que, em ciências sociais e com participantes homogêneos, é comum que o ponto de saturação ocorra até a $12^{\underline{a}}$ entrevista. Estudos empíricos na área também corroboram essa constatação, pois Latham (2013) e Hoffmann et al. (2016) encontraram o ponto de saturação na $11^{\underline{a}}$ entrevista.

O Quadro 6 apresenta um resumo dos seis passos, distintos e complementares, que foram utilizados para a constatação da saturação teórica na pesquisa aqui relatada.

Quadro 6 Passos desenvolvidos para constatação da saturação teórica

\begin{tabular}{l|l}
\hline \multicolumn{1}{c|}{ Passos } & \multicolumn{1}{c}{ Características } \\
\hline 1. Definir Categorias de análise & Selecionar os termos que melhor representam o objetivo central do estudo. \\
\hline 2. Definir o Roteiro de Pesquisa & $\begin{array}{l}\text { Definir as perguntas do roteiro, evitando formulação dúbia dos quesitos, a grande amplitude de res- } \\
\text { postas e o alto grau de variabilidade de diferenciação nas respostas. }\end{array}$ \\
\hline $\begin{array}{l}\text { 3. Definir critérios para a organiza- } \\
\text { ção da amostra }\end{array}$ & $\begin{array}{l}\text { Definir critérios de ordem para os participantes do estudo. Sugere-se que os possíveis participantes da } \\
\text { pesquisa sejam organizados conforme critérios claros que garantam a mesma possibilidade de partici- } \\
\text { pação para qualquer indivíduo que cumpra os requisitos do perfil estabelecido. }\end{array}$ \\
\hline $\begin{array}{l}\text { 4. Levantar elementos novos versus } \\
\text { elementos confirmados em cada } \\
\text { coleta. }\end{array}$ & $\begin{array}{l}\text { Levantar os elementos que são relevantes para o objeto de estudo e classificá-los conforme suas } \\
\text { características }\end{array}$ \\
\hline $\begin{array}{l}\text { 5. Registrar em uma tabela o que foi } \\
\text { encontrado em cada coleta. }\end{array}$ & $\begin{array}{l}\text { Construir uma representação gráfica que permita a visualização dos elementos analíticos que foram } \\
\text { levantados nas entrevistas. }\end{array}$ \\
\hline $\begin{array}{l}\text { 6. Confirmar a saturação em cada } \\
\text { categoria. }\end{array}$ & $\begin{array}{l}\text { Analisar o ponto de saturação, verificar nas últimas entrevistas feitas para confirmação que realmente } \\
\text { não houve novas informações e marcar na representação gráfica feito na etapa anterior onde o ponto } \\
\text { de saturação está para cada categoria. }\end{array}$ \\
\hline
\end{tabular}

Fonte: Elaborado pelos autores.

A técnica de saturação teórica possui procedimentos complexos, como realizar a coleta e a análise dos dados de forma concomitante, mas pode ser adaptada às especificidades de cada trabalho. A saturação, enquanto técnica para definição do número de participantes, pode representar uma boa alternativa para estudos qualitativos que possuem uma população numerosa, e assim inviável de ser entrevistada na sua totalidade, sem fragilizar ou comprometer a 
validade empírica e a credibilidade das análises e dos achados (FONTANELLA et al., 2011).

Dentre as principais dificuldades encontratadas ao aplicar a saturação teórica na investigação a que este artigo faz referência, destacam-se: i) definição do roteiro de pesquisa de forma a evitar grande amplitude nas respostas; ii) administração do tempo, uma vez que um dos pressupostos da técnica é realizar a análise dos dados logo após a coleta. Na saturação teórica é imprescindivel que o investigador tenha finalizado as análises das entrevistas já realizadas antes de voltar a campo. Só assim é possível apurar os elementos que já foram citados e os elementos inéditos; iii) constatação do ponto de saturação. Conforme assinalam Strauss e Corbin (2008), se o investigador procurar com afinco, sempre vai encontrar propriedade ou dimensões adicionais, sempre há potencial para o surgimento do novo. A saturação é mais uma questão de encontrar um ponto na investigação quando coletar dados adicionais torna-se contraprodutivo; o novo que seria revelado não mudaria de forma relevante os resultados já alcançados.

\section{CONSIDERAÇÕES FINAIS}

Determinar o número de entrevistas ou observações que são necessárias é uma questão estratégica para pesquisadores qualitativos. Algumas vezes, o problema não é a quantidade de dados, mas certificar-se que as evidências empíricas coletadas são suficientes para o alcance do objetivo proposto no estudo.

Este trabalho teve como objetivo relatar uma experiência do uso da técnica de saturação teórica no campo das ciências sociais aplicadas, apresentando um roteiro de aplicação e ressaltando os desafios enfrentados em uma pesquisa qualitativa. Acredita-se que o roteiro desenvolvido contribui para o ensino e aplicação futura da técnica. Para o ensino, ao apresentar um roteiro de aplicação sumarizado e, ao ampliar a reflexão junto a investigadores interessados em aprofundar seu conhecimento sobre a saturação teórica, além de apresentar alguns desafios de sua operacionalização já vivenciados. A relevância no sentido da aplicação refere-se ao fato de contribuir com a divulgação de formas de sistematização, tratamento e análise de evidências empíricas em pesquisas qualitativas que utilizem amostras fechadas por saturação.

Destaca-se que para utilizar a técnica é necessário problematizá-la cuidadosamente, uma vez que, a depender dos critérios utilizados, a averiguação de saturação poderá ser realizada de maneira excessivamente subjetiva (STRATUSS; CORBIN, 2008). No roteiro proposto, buscou-se detalhar cada passo, ressaltar as dificuldades vivenciadas e formas possíveis de contorná-las com o objetivo de zelar pelo rigor científico da pesquisa e de auxiliar investigadores qualitativos no uso futuro da técnica.

É possível encontrar na literatura outros estudos que ajudam na operacionalização da saturação teórica. Thiry-Cherques (2009) recomenda um mínimo de oito observações, sendo necessárias duas observações depois de encontrado o ponto de saturação para confirmação. Esse autor também constatou que, em ciências sociais, o ponto de saturação costuma ocorrer até a $12^{\mathrm{a}}$ entrevista. Outro estudo publicado sobre o tema é de Guest, Buncem e Johnson (2006), onde os autores já haviam constatado que a saturação ocorre por volta da $12^{\mathrm{a}}$ entrevista e, além disso, defendem que categorias centrais tendem a aparecer até a $6^{\underline{a}}$ entrevista.

Também é relevante enfatizar que, ao decidir pela utilização da saturação teórica para o fechamento amostral, o investigador precisa ter ciência de que não se pode afirmar com exatidão que novas informações não serão encontradas, o que pode ser interpretado como uma limitação do método, embora não inviabilize a sua confiabilidade. Conforme afirmam Strauss e Corbin (2008), ao procurar com afinco, sempre se encontrarão novas informações. A questão central da saturação teórica é encontrar o momento na coleta de dados em que dados adicionais não mudarão de forma determinante os resultados já alcançados (STRAUSS; CORBIN, 2008).

Como agenda de pesquisa, sugere-se a condução de novos estudos com o emprego da saturação teórica buscando evitar e mitigar as dificuldades aqui relatadas, além de aplicar a técnica em outras áreas de estudo, com vistas a estimular o debate para que o uso da amostragem por saturação em pesquisas qualitativas possa progredir em distintos campos.

Outra oportunidade para avançar no debate sobre o uso da amostragem por saturação está rela- 
cionada à lacuna teórica identificada por O'Reilly e Parker (2012), na qual os autores apontam que estudos que utilizam a saturação teórica quase que exclusivamente se concentram em entrevistas e em grupos focais. Assim, parece existir uma omissão sobre a versatilidade da técnica quando aplicada a outros métodos de coleta de dados, tais como entrada de diários, pesquisa documental, observação participante e ocorrência natural. Essa também é uma oportunidade para promover avanços à pesquisa qualitativa.

\section{REFERÊNCIAS}

ARAÚJO, M. A. D.. Planejamento estratégico: um instrumental à disposição das universidades?. Revista de administração pública, v. 30, n. 4, p. 74-86, 1996.

Bauer, M.; Gaskell, G. (Eds.) Qualitative researching with text, image, and sound. London: Sage, 2008.

CAELLI, K.; RAY, L.; MILL, J.. 'Clear as mud': toward greater clarity in generic qualitative research. International journal of qualitative methods, v. 2, n. 2, p. 1-13, 2003.

CORBIN, J. Grounded Theory. In: Bohnsack, R.; Marotzki w.; Meuser, M. (eds): Haupthegriffe qualitativer Sozialforschung. Opland, 70-75, 2003.

DENZIN, N. K.; LINCOLN, Y. S. O planejamento da pesquisa qualitativa: teorias e abordagens. In: $\mathbf{O}$ planejamento da pesquisa qualitativa: teorias e abordagens. 2006.

DUARTE, R.. Pesquisa qualitativa: reflexões sobre o trabalho de campo. Cadernos de pesquisa, v. 115, n. 1, p. 139-154, 2002.

FALQUETO, J. M. Z. (2012) A implantação do planejamento estratégico em universidades. Dissertação (Mestrado em Administração). Programa de Pós-Graduação em Administração (PPGA/UnB). Universidade de Brasilia, Brasília, Brasil.
FONTANELLA, B. J. B.; LUCHESI, B. M.; SAIDEL, M. G. B.; RICAS, J.; TURATO, E. R;MELO, D. G. Amostragem em pesquisas qualitativas: proposta de procedimento para constatar saturação teórica. Caderno Saúde Pública, v.27, n.2, 389-394, 2011.

FONTANELLA, B. J. B; RICAS, M. G. B.; Turato, J. Amostragem por saturação em pesquisas qualitativas em saúde: contribuições teóricas. Caderno de Saúde Pública, v.24, n.1, 17-27, 2008.

GASKELL, G (2000) Individual and group interviewing. In: Bauer M and Gaskell G (eds). Qualitative researching with text, image and sound. London, Sage, p. 38-56, 2000.

GLASER B. G.; STRAUSS, A. L. The Discovery of Grounded Theory: strategies for qualitative research. Reprinted. New York: Aldine de Gruyter, 2006.

GUEST, G; BUNCE, A.; JOHNSON, L. How many interviews are enough? An experiment with data saturation and variability. Field methods, v. 18, n. 1, p. 59-82, 2006.

HOFFMANN, V. E.; BELUSSI, F. ; MARTÍNEZFERNÁNDEZ, M. T. ; REYES JR., E. . United we stand, divided we fall? Clustered firms' relationships after the 2008 crisis. In: European Academy of Management Conference, Paris. Proceedings of European Academy of Management Conference, 2016. v. 16. p. 1-39.

KAUFMANN, J. C. L’Entretien Compréhensif, Paris : Éditions Nathan, 1996. Disponível em < http://www. unige.ch/fapse/SSE/teachers/maulini/2006/sem-rech-note-lecture.pdf> Acesso em 03 de abril de 2017.

LATHAM, J. R. A framework for leading the transformation to performance excellence part I: CEO perspectives on forces, facilitators, and strategic leadership systems. Quality Management Journal, v. 20, n.2, pp. 22-35, 2013. 
MEYER JÚNIOR, V. Planejamento Universitário: ato racional, político ou simbólico-um estudo de universidades brasileiras. Revista Alcance, v. 12, n. 3 (Set-Dez), p. 373-390, 2008.

MINAYO, M.C.S. O Desafio do Conhecimento: pesquisa qualitativa em saúde. 2.ed., São Paulo: Hucitec/ Abrasco, 1999.

MINAYO, M. C. S. Amostragem e Saturação em Pesquisa Qualitativa: consensos e controvérsias. Revista Qualitativa, v. 5, n. 7 (abril), p. 01-12, 2017.

NEVES, J. L.. Pesquisa qualitativa: características, usos e possibilidades. Caderno de pesquisas em administração, São Paulo, v. 1, n. 3, p. 1-5, 1996.

O'REILLY, M.; PARKER, N.. 'Unsatisfactory Saturation': a critical exploration of the notion of saturated sample sizes in qualitative research. Qualitative Research, v. 13, n. 2, p. 190-197, 2013.

PAIVA JÚNIOR, F. G.; LEÃO, A. L. M. Souza; MELLO, S. C.B. Validade e confiabilidade na pesquisa qualitativa em administração. DOI: 10.5007/2175-8077.2011v13n31p190. Revista de Ciências da Administração, Florianópolis, p. 190209, dez. 2011. ISSN 2175-8077. Disponível em: $<$ https://periodicos.ufsc.br/index.php/adm/article/ view/21758077.2011v13n31p190/20846>. Acesso em: 11 maio 2017.

POZZEBON, M.; FREITAS, H. M. R.; PETRINI, M. Pela integração da inteligência competitiva nos Enterprise Information Systems (EIS). Ciência da informação, v. 26, n. 3, 1997.

PETRINI, Maira; POZZEBON, Marlei. Usando Grounded Theory na construção de modelos teóricos. Gestão \& Planejamento-G\&P, v. 10, n. 1, 2009.

RICHARDSON, R. J. Pesquisa Social: métodos e técnicas. 3. ed. São Paulo: Atlas, 1999.

STRAUSS, A. L.; CORBIN, J. Pesquisa qualitativa: técnicas e procedimentos para o desenvolvimento de teoria fundamentada. Porto Alegre: Artmed, 2008.
SANDELOWSKI, M.. Sample size in qualitative research. Research in nursing \& health, v. 18, n. 2, p. 179-183, 1995.

SANTOS, F. Pesquisa qualitativa: o debate em torno de algumas questões metodológicas. Revista Angolana de Sociologia, n. 14, p. 11-24, 2014.

SOARES, T.; ERDMANN, R. Teoria substantiva em gestão da produção. Revista CADE, v. 12, n. 1, 2013.

THIRY-CHERQUES, H. R. Saturação em pesquisa qualitativa: estimativa empírica de dimensionamento. Revista Brasileira de Pesquisas em Marketing (PMKT), Vol. 3, Setembro, 2009. Disponível em: $<$ http://www.revistapmkt.com.br/Portals/9/Edicoes/ Revista_PMKT_003_02.pdf $>$. Acesso em: 18 de abril de 2017. 